\title{
Finite Element Analysis of Titanium Foam in Mechanical Response for Dental Application
}

\author{
Snehashis Pal ${ }^{1, *}$, Igor Drstvenšek ${ }^{1}$ \\ Faculty of Mechanical Engineering, University of Maribor, Smetanova ulica 17, 2000 Maribor, Slovenia
}

\begin{abstract}
Metals with certain porosity are a new class of materials with extremely low density and a unique combination of excellent mechanical, thermal, electrical, and biocompatible properties. Absorption of impact and shock energy, dust and fluid filtration, construction materials, and most importantly, biocompatible implants are all potential applications for metallic foams. An orthopaedic implant made of metallic foam can provide an open-cell structure that allows for the ingrowth of new bone tissue and the transport of body fluids. Due to its strong biocompatibility and stable fixation between the implant and human bone, titanium foam has recently received much attention as an implant material. Finite element modelling is a suitable method to obtain an efficiently designed implant. Accurate finite element analyses depend on the precision before implementation as well as the functionality of the material properties employed. Since the mechanical performances of titanium foam and solid titanium are different, a constitutive model for porous metal is required. The model of Deshpande and Fleck in the finite element analysis software ABAQUS is used to describe the compressive and flexural deformation properties of titanium foam with $63.5 \%$ porosity. The finite element simulation results were compared with the practical mechanical properties obtained by compression testing of the foam. Finally, the material modelling was used to investigate the stress distributions on the dental implant system.
\end{abstract}

Keywords: finite element analysis; ABAQUS; titanium foam; sintering; dental implant; material modeling; mechanical properties; bending; compressing.

\section{Introduction}

The importance of biological implant fixation for bone and dental applications was recognised more than forty years ago [1]. Metal foams, a new class of materials, are increasingly used in structural components, automotive parts, sound and vibration absorbers, heat exchangers and biomedical implants [2,3]. This is due to their remarkable combination of properties, including low density, high specific stiffness, high specific strength and strong energy absorption [4]. Particularly intriguing is the ability to modify the porosity of cellular materials to match the strength and elastic modulus of bone [5]. Metallic foams have recently undergone a significant improvement in terms of research and development. The requirements for the porous implant are a certain pore morphology, pore size, porosity, and high purity to ensure the biomechanical properties and biocompatibility [6,7]. Methods to produce biocompatible implant metal foams are far from perfect, because the requirements for the porous implant are a certain pore morphology, pore size, porosity and high purity to ensure the biomechanical properties and biocompatibility $[8,9]$.

Titanium foams (Ti foams) are preferred over other metal foams in a number of critical applications, including biomedical implants that must be biocompatible. The main motivation for using cellular metals is to increase the coefficient of friction between the implant and the surrounding bone, which allows mechanical interlocking 
of the bone with the implant component through significant bone ingrowth and good stability [10]. Additionally, to reduce the stress shielding effect, the stiffness of the implants can be adjusted by varying the porosity of the implants [11-13]. The present study addresses one of these possibilities, a successful biomedical application of Ti foams in a dental implant system.

The use of simulation tools for finite element analysis has played a crucial role in the development of lightweight cellular structures [14-16] as well as dental implants [17]. The accuracy and efficiency of the material models used for dental implants depends on the use of finite element method to analyse structures and components. Porous titanium has slightly different mechanical properties than solid titanium when subjected to loading. Solid titanium is an incompressible material whose volume does not change during deformation, whereas titanium with a porous structure is a compressible material whose volume changes during deformation. Consequently, porous metal yields under both hydrostatic loading and deviatoric loading. In Ti foam, different forms of porosity lead to different mechanical properties and failure mechanisms $[18,19]$. To simulate metal foam using finite element method, an effective metal foam constitutive model is needed. In the last decade, finite element packages such as LS -DYNA and ABAQUS have been used to build constitutive models for metal foams $[20,21]$. However, few simulations of Ti foam under complex loading conditions have been studied and reported. The aim of this study was to examine whether the model of Deshpande and Fleck, which is included in the ABAQUS finite element technique as a material model for crushable foams, can be used to describe the properties of Ti foam. Furthermore, the stress distribution on Ti foam for dental implants subjected to complex stress conditions is investigated.

\section{Experimental}

\subsection{Mechanical Properties of Metallic Foams}

Metal foams have a large number of cells or pores, similar to a sponge, which distinguishes them from solid metals. The cell topology, relative density, cell size and cell shape of a metal foam are defined microstructurally [22]. At the macroscopic scale, the term porosity is used to describe the fraction of porous area in foams. The mechanical properties of cellular metals are affected by their microstructure [23]. There are three regimes in the stress-strain curve of a metal foam in compression. One is the linear elastic law, which corresponds to the bending of the cell or the stretching of the surface. The second law is the stress plateau rule, which corresponds to progressive cell collapse caused by elastic buckling or plastic yielding. The third law is the densification rule, which corresponds to the collapse of cells in the material and the resulting stacking of cell edges and faces against each other. Low density metal foam, on the other hand, can be deformed to a significant strain before compaction [24]. For effective applications as functional parts, understanding the plastic yield point and subsequent plastic flow behaviour of a metal foam is critical. Metal foams can yield under both hydrostatic and deviatoric loading compared to solid metals [4]. As a result, both the von Mises equivalent stress and the mean stress influence the yield criterion.

\subsection{Sintering of titanium foam}

The powder metallurgy space holder technique was used to produce porous titanium monoliths. The titanium ( $\mathrm{Ti}$ ) powder with a particle diameter range between $50 \mu \mathrm{m}$ and $100 \mu \mathrm{m}$ was provided by Micro\&Polo, Slovenia. The ammonium bicarbonate powder with a particle diameter of about $200 \mu \mathrm{m}$, provided by Sigma-Aldrich, Germany, was used as a space holder during green body formation. Evaporation of ammonium bicarbonate, which has almost no strong solubility in titanium, was used to produce open-pore structures. Titanium powder at 60 vol\% was homogeneously mixed with carbamide at 40 vol\% for 15 minutes. While mixing the powder, a binder, polyvinyl alcohol (PVA) with $99+\%$ hydrolysis from Sigma-Aldrich, Germany, was used in an amount of $5 \mathrm{wt} \%$. The green bodies were then produced using $500 \mathrm{MPa}$ unilateral pressure under a cold compression technique in a Manual Hydraulic Press - KBr pelletizer. The equipment had a $13 \mathrm{~mm}$ diameter cylindrical powder hopper with a lower punch that served as a rigid base and an upper punch that applied uniaxial pressure. The samples were heat treated in a tube furnace under argon atmosphere to burn out the binder and sinter the porous Ti monoliths. The heat treatment consisted of two steps: first heating to $550^{\circ} \mathrm{C}$ at a rate of $5{ }^{\circ} \mathrm{C} / \mathrm{min}$ for 30 minutes to burn out the binder, and then heating to $950{ }^{\circ} \mathrm{C}$ at the same rate for 2 hours. The test specimens were cooled to room 
Figure 1: Both wire mechanism variants (basic position).

temperature in the oven. The resulting total porosity (P in \%) of the porous titanium foams was calculated using equation (1). Here, $\rho$ s denotes the density of $\mathrm{Ti}$ and $\rho$ denotes the density of the porous $\mathrm{Ti}$ specimen, which is determined as the volume-tomass ratio.

$$
P=\left(1-\frac{\rho}{\rho_{s}}\right) \times 100
$$

However, the authors have explored the compressive mechanical properties of different ratios of Ti and carbamate where 50 vol\%, 60 vol\%, and 70 vol\% Ti were taken into account. The best Tifoam have been selected considering the enough strength bearing and existence of sufficient porosity.

\subsection{Material Modelling for Metallic Foams}

Classical plastic theory cannot be used to explain such behaviours because the mechanical properties of metal foam differ from those of solid metal. Several constitutive models have yet to be established. The yield point has been shown to depend on both the mean stress and the von Mises equivalent stress, and both models are phenomenological.

The yield surface is a Mises circle in the deviatoric stress plane and an ellipse in the meridional $(p-q)$ stress plane for the plastic part of the mechanical action. Deshpande and Fleck [25] created a 3D model based on Al foam experiments designed with a finite element package. ABAQUS is an acronym for ABAQUS Deshpande and Fleck created the phenomenological isotropic model for metallic foams (2000). The uniaxial compressive yield stress as a function of the corresponding plastic strain must be defined by the strain hardening curve. In defining this dependence at finite strains, the "true" (Cauchy) stress and log strain values should be given. For pressure-dominated loading, both models predict similar effects.

The volumetric strain hardening model, on the other hand, assumes perfectly plastic behaviour under hydrostatic tension, while the isotropic strain hardening model predicts the same behaviour under both hydrostatic tension and hydrostatic compression. The contribution of mean stress to the yield function in the crushable foam model is realized by a material parameter known as the shape factor, which determines the aspect ratio of the elliptical stress. This shape factor is used to quantitatively distinguish metal foams from solid metals in terms of their plastic properties. The yield function $(F)$ is provided as equation (2).

$F=\sqrt{q^{2}+\alpha^{2}\left(p-p_{0}\right)^{2}}-B$

The von Mises equivalent stress is $q$, and the mean stress is $p$. The yield ellipse's size is defined by $B$, which is defined in equation (3).

$B=\alpha p_{c}=\sqrt{1+\left(\frac{\alpha}{3}\right)^{2}}$

In hydrostatic compression, $p_{c}$ denotes the yield strength. The absolute value of the yield strength in uniaxial compression is denoted by $\sigma_{c}$. The yield surface's shape factor $(\alpha)$ can be presented as the equation (4). The ratio of the initial yield stress in uniaxial compression and the initial yield stress in hydrostatic compression is presented by $k$.

$\alpha=\frac{3 k}{\sqrt{9-k^{2}}}$

As shown in Fig. 1, equation (2) describes an elliptical yield surface in the stress plane of von Mises stress versus mean stress.

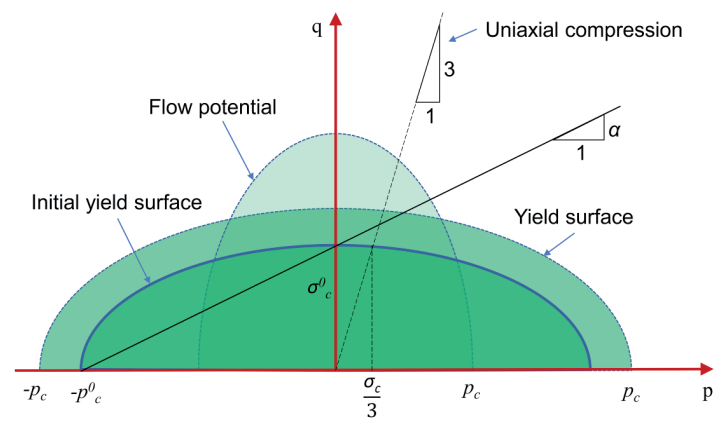

Figure 1: Crushable foam model with volumetric hardening: yield surface and flow potential in the $p-q$ stress plane.

The isotropic hardening model's flow potential is set as

$G=\sqrt{q^{2}+\beta^{2} p^{2}}$

$\beta=\frac{3}{\sqrt{2}} \sqrt{\frac{1-2 v_{p}}{1+v_{p}}}$

The plastic Poisson's ratio vp can be as the equation (7) 
$v_{p}=\frac{3-k^{2}}{6}$

The plastic strain $\left(\varepsilon^{p l}\right)$ is as equation (8) where $\lambda$ denotes the nonnegative plastic flow multiplier. Only linear elastic behaviour can be used to model the elastic property. The Young's modulus Ef for metal foam, which is based on foam porosity, must be used.

$\varepsilon^{p l}=\frac{\partial G}{\partial}$

\subsection{Selection of Ti-foam Material Parameters}

The Young's modulus (E), the Ti-foam yield strength $\left(\sigma_{y}\right)$, the shape factor $(\alpha)$, the compressible yield stress ratio $(k)$, the elastic and plastic Poisson's ratio $\left(v, v_{p}\right)$, and the hardening relation are all needed material parameters for the crushable foam model. To represent the shape of the workpiece during deformation, the plastic Poisson's ratio was chosen. The parameters $k$ is calculated using equation (4) and $\alpha$ is determined from using equation (7). For initial calibration and validation of the crushable foam model, the strategy was to use information from Imwinkelried's experimental results [25]. The model was then used to predict the mechanical behaviour of Ti-foam during compression and bending at different levels of porosity.

\section{Results and Discussion}

\subsection{Finite Element Analysis of Compression Tests}

To assess the accuracy of the crushable foam model in capturing the mechanical behaviour of Tifoam, a compression test of a $63.5 \%$ porosity Ti-foam cylinder with $13 \mathrm{~mm}$ diameter and $15 \mathrm{~mm}$ height was simulated as shown in Fig. 2. The upper flat die was subjected to a $10 \mathrm{kN}$ force, while the lower flat die was left alone. Rigid surfaces were used to model the dies. For the cylindrical workpiece, axisymmetric elements were used. The contact surfaces were given a friction coefficient of 0.5 [26]. The ultimate tensile strength, yield strength and strain up to break of the T-foam have been obtained using the compressive strength tests and those are $16.9 \mathrm{MPa}$, $11 \mathrm{MPa}$, and $31 \%$ respectively.

The yield stress $\left(\sigma_{y}\right)$ and Young modulus (E) of $63.5 \%$ porosity Ti-foam were determined using (9) and (10) respectively those are obtained from Imwinkelried's experiments [25].

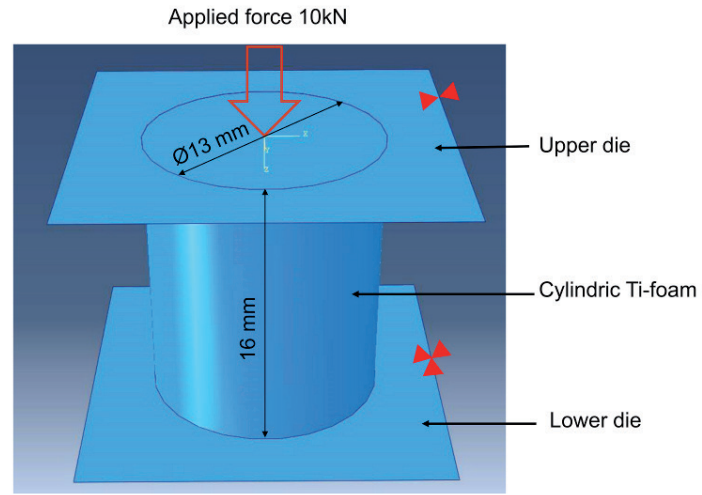

Figure 2: Cylindrical specimen model in ABAQUS for simulations of compression tests.

$E=E_{s}\left(-0.024+15.86 \rho_{r}^{2}\right)$

$\sigma_{y}=\left(\sigma_{y}\right)_{s}\left(-0.068+7.684 \rho_{r}^{\frac{3}{2}}\right)$

Here Es denotes the fully solid titanium's Young's modulus, which is $110 \mathrm{GPa}$ for pure titanium grade 4 [25]. Whereas, $\left(\sigma_{y}\right)$, denotes the yield strength for the fully solid titanium which is $650 \mathrm{MPa}$. The relative density of the metal foam is represented by pr. According to Imwinkelried's experimental results, it is linearly proportional to percent porosity. To represent the shape of the specimen, the plastic Poison's ratio $\left(v_{p}\right)$ was assumed to be 0.34 . From equation (6), the compressible yield stress ratio ( $k$ ) was found to be 0.98 . Fig. 3 shows a contour plot of von Mises tension of compressed Ti-foam. Total number of nodes is 3504 and total number of elements is 2955 with 2955 linear hexahedral elements of type C3D8R.

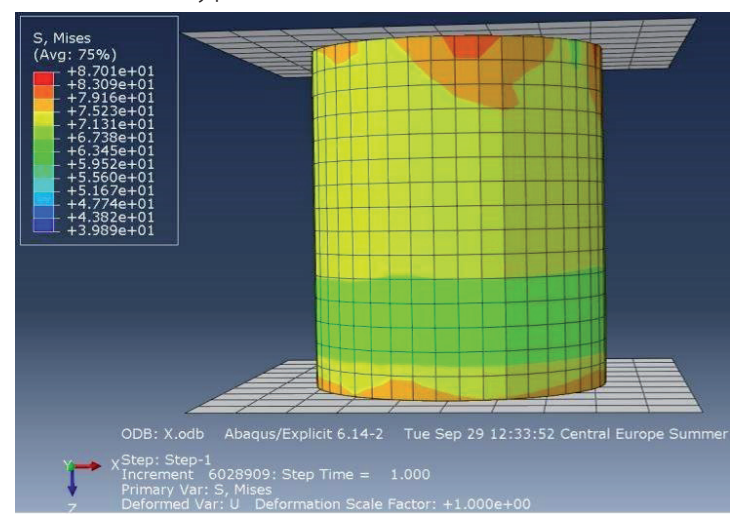

Figure 3: The von Mises stress distribution on the compressed specimen.

The yield stress for Ti-foam with 63 percent porosity is 70 to $80 \mathrm{MPa}$. Assume a concentrated 
force of $10 \mathrm{kN}$, and very few places have a stress result of about 70 to 90 . So, the permissible results can be achieved for dental applications having the load of $17.1 \mathrm{~N}, 114.6 \mathrm{~N}$, and $23.4 \mathrm{~N}$ in the lingual, axial, and mesio-distal directions [27]. Fig. 4 depicts the displacement plot in compression of the specimen.

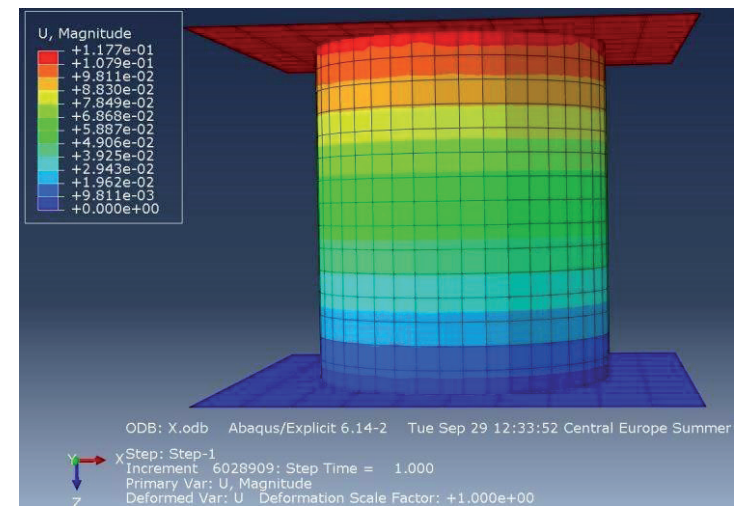

Figure 4: Displacement plot in compression of the compressed specimen.

\subsection{Finite Element Analysis of Three-point bending Test}

Using the same material model used in the compression tests, a three-point bending test of Ti foam with $63.5 \%$ porosity was simulated for comparison with the bending test of Imwinkelried [25]. Fig. 5 shows a simulation of the three-point bending test model. Steel sheet bodies were used to model two support rollers and the upper movable roller. Two support rollers were installed with a distance of $72 \mathrm{~mm}$ gap between them. The width and height of the specimen were $20 \mathrm{~mm}$ and $5 \mathrm{~mm}$, respectively. The upper movable roller was lowered to a depth of $5 \mathrm{~mm}$. C3D20R, the standard three-dimensional standard quadratic hexahedral elements, were used to mesh the specimen. The total number of nodes was 88901, with a total number of 20000 elements.

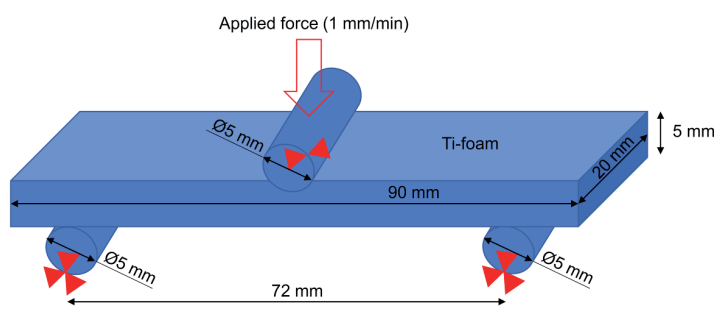

Figure 5: The model set up in ABAQUS for the three-point bending test.
Fig. 6 depicts the deformed shape of a Ti foam specimen with a $63.5 \%$ porosity under a 3 -point bending load. It is clear that the stress is localized, with the highest equivalent stress occurring near the bending region. The model of Deshpande and Fleck used to define the properties of the Ti deformation foam was calibrated and a comparative analysis was performed with the experimental results of Imwinkelried [25]. The results are in broad agreement. The total stress at the outer surface of the beam is zero, and the stresses at the centroidal position are balanced. If one thinks about it, this finding is obvious since the stresses above and below the $X$-axis of the beam, which is in compression and below the axis, which is in tension, increase at the same linear rate. Bending performance for bone implantation has shown excellent results $[28,29]$.

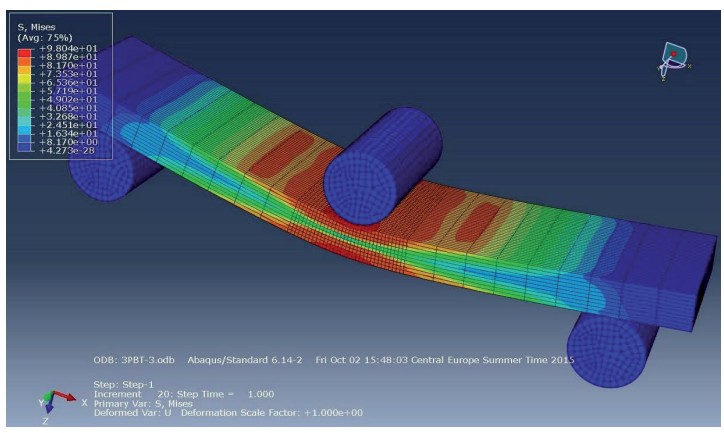

Figure 6: Contour plot of von Mises stress on the bending specimen.

\subsection{Application of Ti-Foam Model on Dental Implant}

For the purpose of FEM simulation, a simple model was used in this study. The use of fully porous titanium has the ability to solve fracture and stress shielding problems in bone $[11,12]$. A threedimensional finite element analysis for a Ti foam dental implant system was performed to investigate the feasibility of using Ti foam as a dental implant. Fig. 7 portrays a three-dimensional simple model of a Ti foam dental implant and a supporting bone system.

An implant and the underlying cancellous and cortical tissues form the dental implant model. In Fig. 7, the implant and cortical bone is visible, however, the cancellous bone existed in between them and inside the cortical bone. All rotational and translational movements in the model were constrained by the boundary conditions. The interface between the implant and the bone and 
between the cortical bone and the cancellous bone was assumed to be perfect. Fig. 7 shows that the implant is loaded in the lingual, axial, and mesiodistal directions with forces of $17.1 \mathrm{~N}, 114.6 \mathrm{~N}$, and 23.4 $\mathrm{N}$, respectively $[26,27]$. 3D solid continuum components were used to construct the finite element mesh. Fig. 8 shows how the shape of the implant was simplified.

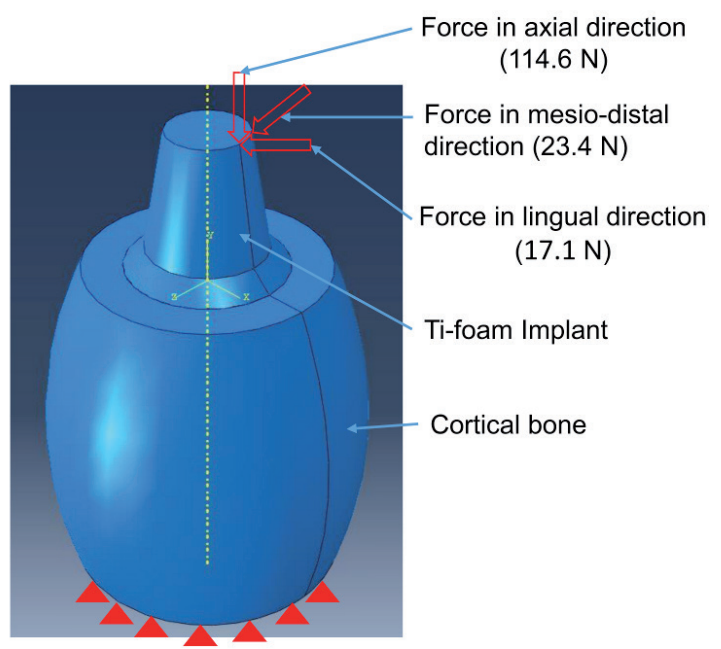

Figure 7: ABAQUS model of a dental implant having surrounding bone system with applied loads and boundary conditions.

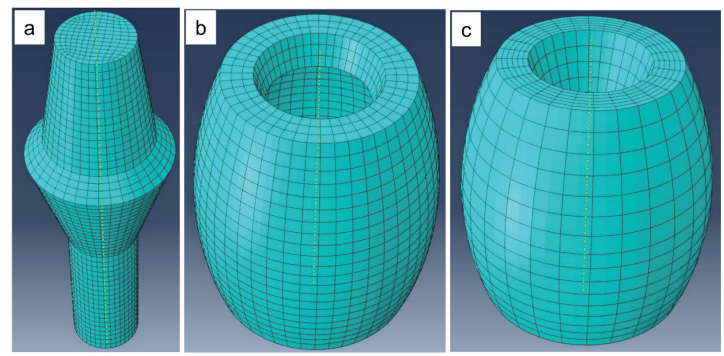

Figure 8: The finite element mesh of (a) Implant part, (b) Cortical Bone, and (c) Cancellous Bone.

The tests were performed on the porous titanium implant. The stress distribution is uniform around the implants. Fig. 9 shows that the highest equivalent stresses in the implants are concentrated on the neck side. 7700 quadratic hexahedral elements of form C3D20R were used to mesh the part, and the total number of nodes was 34671.

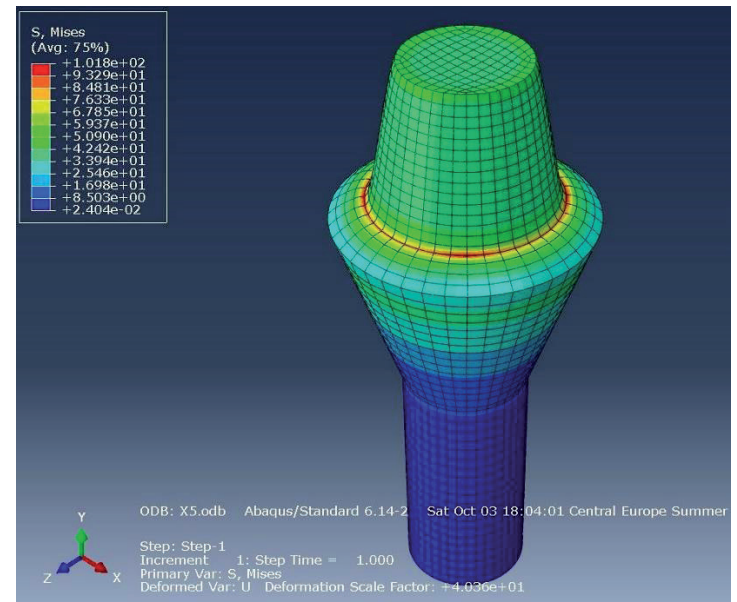

Figure 9: The Ti-foam implant with von Mises stresses distribution within it.

\section{Conclusions}

To explain the behaviour of the porous structure of titanium, the model of Deshpande and Fleck was used, which is available in the finite element simulation program ABAQUS as a model for crushable foam. The required material properties were chosen based on Imwinkelried's experimental results. The model was calibrated, and a comparative analysis with experimental data with a porosity of 63.5\% was performed for both compression and bending, with acceptable agreement.

Fairly great agreement was obtained in these tests. The dental implant device was simulated using a crushable foam model with calibrated parameters. In this test, it can be seen that the surrounding bone of the Ti foam implant system was subjected to further stresses. This means that more stresses were transferred to the surrounding bone when the Ti foam implant was used. As a result, the stress shielding problem can be solved.

This is an abbreviated analysis to demonstrate the applicability of Deshpande and Fleck's model to the Ti foam implant system. For future application, a thorough investigation of the design and optimization of the Ti foam implant and the dental implant system is required.

\section{Acknowledgments}

The authors acknowledgethe financial support from the Slovenian Research Agency (Research Core Funding No. P2-0157, Research Project J1-2470 Biofunctionalization of 3D-printed metal alloys as a newly emerging strategy to diminish undesired effects of orthopedic implants, Research project J3-9262 Advanced surface 
finishing technologies for antibacterial properties of patientspecific 3D-printed implantable materials). This project has also received funding from the European Union's Horizon 2020 research and innovation program under grant agreement No. 788361.

\section{References}

1. Bobyn, J.D.; Pilliar, R.M.; Cameron, H.U.; Weatherly, G.C.; Kent, G.M. The effect of porous surface configuration on the tensile strength of fixation of implants by bone ingrowth. Clinical Orthopaedics and Related Research 1980, NO 149, 291-298.

2. Vendra, L.; Rabiei, A. Evaluation of modulus of elasticity of composite metal foams by experimental and numerical techniques. Materials Science and Engineering A 2010, 527, 1784-1790

3. Tuncer, N.; Arslan, G. Designing compressive properties of titanium foams. Journal of Materials Science 2009, 44, 1477-1484.

4. Ashby, M.; Evans, A.; Fleck, N.; Gibson, L.; Hutchinson, J.; Wadley, H.; Delale, F. Metal Foams: A Design Guide. Applied Mechanics Reviews 2001, 54, B105-B106.

5. Wen, C.E.; Mabuchi, M.; Yamada, Y.; Shimojima, K.; Chino, Y.; Asahina, T. Processing of biocompatible porous Ti and $\mathrm{Mg}$. Scripta Materialia 2001,45, 1147-1153.

6. Nouri, A.; D., P.; We, C. Biomimetic Porous Titanium Scaffolds for Orthopedic and Dental Applications; InTech: Australia, Australia/Oceania, 2010;

7. Gómez, S.; Vlad, M.D.; López, J.; Fernández, E. Design and properties of 3D scaffolds for bone tissue engineering. Acta Biomaterialia 2016, 42, 341-350.

8. Tiyyagura, H.R.; Mohan, T.; Pal, S.; Mohan, M.K. Surface modification of Magnesium and its alloy as orthopedic biomaterials with biopolymers. In Fundamental Biomaterials: Metals; Elsevier, 2018; pp. 197-210 ISBN 9780081022054.

9. Bružauskaitè, I.; Bironaitè, D.; Bagdonas, E.; Bernotienè, E. Scaffolds and cells for tissue regeneration: different scaffold pore sizes_-different cell effects. Cytotechnology 2016, 68, 355-369.

10. Pal, S.; Peršin, Z.; Vuherer, T.; Drstvenšek, I.; Kokol, V. The Effect of Ti-6Al-4V Alloy Surface Structure on the Adhesion and Morphology of Unidirectional Freeze-Coated Gelatin. Coatings 2020, 10, 434.

11. Ridzwan, M.I.Z.; Shuib, S.; Hassan, A.Y.; Shokri, A.A.; Mohammad Ibrahim, M.N. Problem of stress shielding and improvement to the hip implant designs: A review. Journal of Medical Sciences 2007, 7, 460-467.

12. Yan, W.; Pun, C.L. Spherical indentation of metallic foams. Materials Science and Engineering A 2010, 527, 3166-3175.
13. Pal, S.; Tiyyagura, H.R.; Drstvenšek, I.; Kumar, C.S. The Effect of Post-processing and Machining Process Parameters on Properties of Stainless Steel PH1 Product Produced by Direct Metal Laser Sintering. Procedia Engineering 2016, 149, 359-365.

14. Ramadani, R.; Belsak, A.; Kegl, M.; Predan, J.; Pehan, S. Topology Optimization Based Design of Lightweight and Low Vibration Gear Bodies. International Journal of Simulation Modelling 2018, 17, 92-104.

15. Ramadani, R.; Kegl, M.; Predan, J.; Belšak, A.; Pehan, S. Influence of cellular lattice body structure on gear vibration induced by meshing. Strojniski Vestnik/Journal of Mechanical Engineering 2018, 64, 611-620.

16. Ramadani, R.; Pal, S.; Kegl, M.; Predan, J.; Drstvenšek, I.; Pehan, S.; Belšak, A. Topology optimization and additive manufacturing in producing lightweight and low vibration gear body. The International Journal of Advanced Manufacturing Technology 2021. https://doi.org/10.1007/ s00170-021-06841-W

17. Merdji, A.; Bachir Bouiadjra, B.; Achour, T.; Serier, B.; Ould Chikh, B.; Feng, Z.O. Stress analysis in dental prosthesis. Computational Materials Science 2010, 49, 126-133.

18. Schiefer, H.; Bram, M.; Buchkremer, H.P.; Stöver, D. Mechanical examinations on dental implants with porous titanium coating. Journal of Materials Science: Materials in Medicine 2009, 20, 1763-1770.

19. Kashef, S.; Asgari, A.; Hilditch, T.B.; Yan, W.; Goel, V.K.; Hodgson, P.D. Fracture toughness of titanium foams for medical applications. Materials Science and Engineering A 2010, 527, 7689-7693.

20. Deshpande, V.S.; Fleck, N.A. Isotropic constitutive models for metallic foams. Journal of the Mechanics and Physics of Solids 2000, 48, 1253-1283.

21. Reddy, T.H.; Pal, S.; Kumar, K.C.; Mohan, M.K.; Kokol, V. Finite element analysis for mechanical response of magnesium foams with regular structure obtained by powder metallurgy method. Procedia Engineering 2016, 149, 425430.

22. Nouri, A.; Chen, X.B.; Hodgson, P.D.; Wen, C.E. Preparation and characterisation of new titanium based alloys for orthopaedic and dental applications. Advanced Materials Research 2007, 15-17, 71-76.

23. Pal, S.; Lojen, G.; Gubeljak, N.; Kokol, V.; Drstvensek, I. Melting, fusion and solidification behaviors of Ti-6Al-4V alloy in selective laser melting at different scanning speeds. Rapid Prototyping Journal 2020, 26, 1209-1215.

24. Wolcott, M.P. Cellular solids: Structure and properties. Materials Science and Engineering: A 1990, 123, 282-283.

25. Imwinkelried, T. Mechanical properties of open-pore titanium foam. Journal of Biomedical Materials Research - 
Part A 2007, 81, 964-970

26. Kayabaşi, O.; Yüzbasioğlu, E.; Erzincanli, F. Static, dynamic and fatigue behaviors of dental implant using finite element method. Advances in Engineering Software 2006, 37, 649-658.

27. Djebbar, N.; Serier, B.; Bouiadjra, B.B.; Benbarek, S.; Drai, A. Analysis of the effect of load direction on the stress distribution in dental implant. Materials and Design 2010, 31, 2097-2101.
Shih, K.-S.; Hsu, C.-C.; Hou, S.-M.; Yu, S.-C.; Liaw, C.-K. Comparison of the bending performance of solid and cannulated spinal pedicle screws using finite element analyses and biomechanical tests. Medical Engineering \& Physics 2015, 37, 879-884.

29. Ridha, H.; Thurner, P.J. Finite element prediction with experimental validation of damage distribution in single trabeculae during three-point bending tests. Journal of the Mechanical Behavior of Biomedical Materials 2013, 27, 94-106.

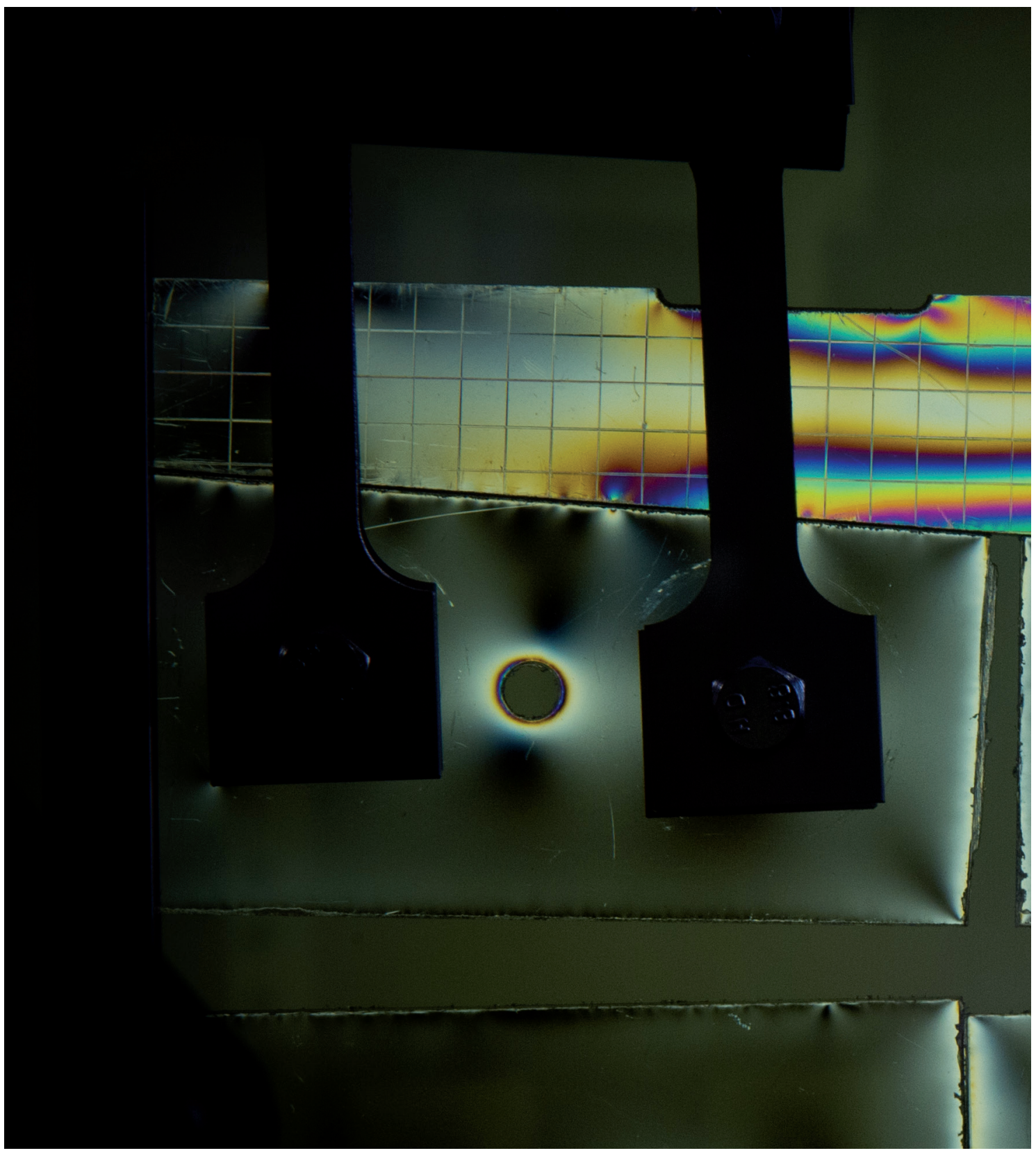

